\title{
A mathematical evaluation for measuring correctness of domain ontologies using concept maps
}

\begin{abstract}
There is a need for further research in the area of ontology evaluation specifically dealing with ontology development exploiting concept maps. The existing literature on ontology evaluation primarily emphasis on ontology formalisation as well as on performing logical inferences, which is usually not directly relevant for concept maps as they are commonly exploited as communication instruments for learning purposes. Commonly used techniques for evaluating concept maps for knowledge assessment may be adopted for a kind of criteria-based evaluation of a domain concept map with respect to a particular aspect. However, this makes its validity limited to a particular aspect or criteria. This paper presents a mathematical ontology evaluation technique to measure the correctness of domain ontologies engineered using concept maps. It is based on the notion of merging two different mathematical measures, namely closeness index and similarity index to come up with a combined index that takes different criteria or aspects into account while performing ontology evaluation. Therefore, the proposed technique makes the evaluation process more reliable and robust. Two case studies were conducted employing the proposed technique for evaluating two different domain ontologies that were engineered using concept maps. Calculations and results from the case studies showed that depending on the correctness of individual ontology, different values of combined Index was calculated manifesting the measure of correctness of each individual ontology in a quantifiable form. Moreover, the results depict that the technique provides in-depth evaluation, it is easy to adopt, requires no special skills, and is conveniently replicable.
\end{abstract}

Keyword: Ontology engineering; Concept mapping; Ontology evaluation; Closeness index; Similarity index 